\title{
IDOSOS COM DEPRESSÃO: UMA ANÁLISE DOS FATORES DE INSTITUCIONALIZAÇÃO E APOIO FAMILIAR
}

\author{
Depressed older adults: an analysis of factors related to institutionalization and \\ family support
}

\section{Mayores con depresión: un análisis de los factores de la institucionalización y del apoyo familiar}

Maria Vieira de Lima Saintrain

Universidade de Fortaleza - UNIFOR - Fortaleza (CE) - Brasil

Carina Barbosa Bandeira

Universidade de Fortaleza - UNIFOR - Fortaleza (CE) - Brasil

Marina Arrais Nobre

Universidade de Fortaleza - UNIFOR - Fortaleza (CE) - Brasil

Rafaela Lais Pesenti Sandrin

Universidade de Fortaleza - UNIFOR - Fortaleza (CE) - Brasil

\section{RESUMO}

Objetivo: Identificar a prevalência de depressão de idosos residentes em instituições de longa permanência (ILPI) e sua relação com os motivos de institucionalização. Métodos: Estudo quantitativo e transversal realizado em Fortaleza, Ceará, que utilizou um instrumento de coleta de dados elaborado para realizar avaliação clínica concernente à depressão maior, utilizando os critérios diagnósticos do Diagnostical and statistical manual of mental disorders (DSM-IV-TR). Serviu também para registrar os dados secundários obtidos dos prontuários dos pacientes idosos no intuito de complementar as informações relativas ao contexto da institucionalização. Resultados: Do total de idosos pesquisados, 82 (34,6\%) apresentaram diagnóstico de Depressão Maior segundo os critérios do DSM-IV-TR. No diagnóstico de depressão por ILPI, verifica-se significância estatística entre as duas ILPI $(p=0,042)$. Na associação verificada entre depressão e tempo de institucionalização $(p=0,001)$, é importante destacar o maior percentual entre os idosos com até três anos de institucionalização ( $37,8 \%$ dos casos de depressão), levando se a considerar que o pouco tempo de afastamento familiar e o processo de adaptação ao novo tipo de moradia possam constituir fatores de risco para a doença. Conclusão: Os achados acima descritos aludem à ideia de que o tempo de institucionalização, a carência das relações interpessoais, a solidão e o fato de o idoso receber visitas de familiares neste período constituem fatores de risco para a depressão.

Descritores: Depressão; Idoso; Institucionalização.

\section{ABSTRACT}

Objective: To identify the prevalence of depression in older adults living in long-term care (LTC) facilities and its association with the reasons for institutionalization. Methods: Quantitative, cross-sectional study carried out in Fortaleza, Ceará, using a data collection instrument designed to perform clinical assessment of major depression, based on the Diagnostical and statistical manual of mental disorders (DSM-IV-TR) diagnostic criteria. It was also used to record secondary data obtained from the medical records of the older adult patients in order to complement information on the institutionalization context. Results: Of all older adults analyzed, 82 (34.6\%) presented a diagnosis of major depression according to DSM-IV-TR criteria. As for the diagnosis of depression related to the LTC facility, there was a statistically significant difference between the two LTC facilities ( $p=0.042$ ). With regard to the association between depression and institutionalization duration $(p=0.001)$, it is important to highlight a higher percentage among older adults with up to three years of institutionalization (37.8\% of the cases of depression), which suggests that a short period of separation from family and the process of adaptation to the new type of housing may constitute risk factors 
for the disease. Conclusion: The findings described above allude to the idea that institutionalization duration, lack of interpersonal relationships, loneliness and the fact that the older adults receive visits from family members in this period are risk factors for depression.

Descriptors: Depression; Aged; Institutionalization.

\section{RESUMEN}

Objetivo: Identificar la prevalencia de depresión de mayores que viven en instituciones de larga permanencia (ILP) y su relación con los motivos de la institucionalización. Métodos: Estudio cuantitativo y transversal realizado en Fortaleza, Ceará, que utilizó un instrumento para la recogida de datos que ha sido elaborado para realizar la evaluación clínica de la depresión mayor utilizando los criterios diagnósticos del Diagnostical and statistical manual of mental disorders (DSM-IV-TR). Ha servido también para registrar los datos secundarios de los historiales clínicos de los pacientes mayores para complementar las informaciones relativas al contexto de la institucionalización. Resultados: Del total de mayores investigados, $82(34,6 \%)$ presentaron el diagnóstico de Depresión Mayor según los criterios del DSM-IV-TR. En el diagnóstico de depresión por ILP se verifica la significación estadística entre las dos ILP $(p=0,042)$. En la asociación verificada entre la depresión y el tiempo de institucionalización ( $p=0,001)$ se puede destacar el mayor porcentual entre los mayores de hasta tres años de institucionalización (37,8\% de los casos de depresión) considerándose que el poco tiempo de alejamiento familiar y el proceso de adaptación al nuevo tipo de vivienda pueden ser factores de riesgo para la enfermedad. Conclusión: Los hallazgos descritos indican la idea de que el tiempo de institucionalización, la carencia de las relaciones interpersonales, la soledad y el hecho del mayor recibir visitas de los familiares en ese periodo son factores de riesgo para la depresión.

Descriptores: Depresión; Anciano; Institucionalización.

\section{INTRODUÇÃO}

O início da transição demográfica de uma população com mais crianças para outra com mais idosos teve início na Europa. Paralelamente, o progresso da ciência da saúde permitiu a maior sobrevida da população, sobretudo em países desenvolvidos, nos quais a definição de idoso passou a ser de pessoas com 65 anos ou mais, e nos países em desenvolvimento permanece a definição anterior de 60 anos ou mais. O número de idosos aumentou 48\% só em 2015 e prevê-se que terá triplicado em 2050 em relação ao ano 2000, segundo o The World Population Ageing Report ${ }^{(1)}$.

A alteração do perfil etário da população implica em aumento da prevalência de doenças crônicas, necessitando de aprimoração das ações relacionadas à prevenção de doenças, uma vez que na maioria dos países as políticas públicas em saúde são voltadas para a resolução de problemas agudos, incluindo-se as doenças de curso crônico ${ }^{(2-4)}$. Desta feita, é necessária a ampliação dos estudos para detecção de fatores de risco para tais enfermidades, objetivando melhora na qualidade das ações de promoção de saúde e prevenção de doenças na comunidade.

Nesse contexto, uma das principais doenças crônicas que atingem a população envelhecida é a depressão maior $^{(5)}$, prejudicando a funcionalidade do idoso e tornando-se um grave problema de saúde pública, uma vez que há prejuízo na qualidade de vida e aumento nos gastos com serviços de saúde, além de, na maioria das vezes, ser este um problema que envolve também a família, pois os idosos se tornam dependentes.

Tem sido observado que a depressão maior nos idosos se manifesta de forma diferente das outras faixas etárias ${ }^{(5)}$, principalmente no que diz respeito às diferenças de sintomatologia, devendo-se levar em conta as particularidades próprias da idade ${ }^{(6)}$. Entre as principais variáveis associadas à depressão, encontram-se idade avançada, sexo feminino, condições de saúde, como a incapacidade funcional, doenças crônicas e déficit cognitivo, podendo ser a institucionalização uma situação estressante e desencadeadora de depressão(7).

Paralelamente, uma das maiores peculiaridades dos idosos é a ideia de aproximação do fim da vida, que os tornam mais suscetíveis a sentimentos de solidão e isolamento, o que pode resultar de eventos que diminuem a funcionalidade ou o impacto aprimorado do luto( ${ }^{(8)}$, levando à maior incidência de depressão em idosos ${ }^{(9)}$. Em suma, a solidão é um preditor de severidade da depressão( ${ }^{(10)}$.

Estima-se que $15 \%$ dos idosos exprimem alguma sintomatologia depressiva, sendo $2 \%$ deles na forma mais grave. Em algumas populações, como em idosos hospitalizados ou institucionalizados, a frequência é maior, atingindo de $5 \%$ a $13 \%$ dos pacientes hospitalizados e $12 \%$ a $16 \%$ dos residentes em instituição de longa permanência (IPLI) para idosos. Aproximadamente $13 \%$ dos idosos que vivem em IPLI desenvolvem episódio depressivo dentro de 
um ano após sua institucionalização(11). Estudo apontou que idosos residentes em moradia específica para idosos apresentaram chance 2,7 vezes maior de apresentar depressão do que os residentes na comunidade ${ }^{(12)}$.

Nesse contexto, as famílias têm grande impacto nos cuidados de saúde de idosos, seja na adesão ao tratamento ou na progressão da doença, principalmente na depressão, podendo causar mudanças comportamentais em longo prazo, de modo que o apoio social é preditor independente de desfechos de depressão geriátrica. Os adultos jovens com apoio familiar positivo têm menores chances de serem institucionalizados, vista que a ausência de cuidados constitui um dos principais motivos de institucionalização. Entretanto, enquanto o apoio familiar positivo seja protetor para o paciente, a hostilidade familiar é um preditor de pior evolução da doença, acarretando em maior taxa de suicídios $^{(13,14)}$.

Diante desta problemática a pesquisa teve como objetivo identificar a prevalência de depressão em idosos residentes em instituições de longa permanência e sua relação com os motivos de institucionalização.

\section{MÉTODOS}

Trata-se de um estudo quantitativo e transversal no qual se utilizou um instrumento de coleta de dados elaborado para realizar avaliação clínica concernente à depressão maior. Considerando o diagnóstico de depressão, além da história clínica e dos dados obtidos no exame do estado mental, são utilizados sistemas classificatórios que relacionam os sintomas clínicos para a realização do diagnóstico, utilizando-se, portanto, nesta pesquisa os critérios diagnósticos do Diagnostical and statistical manual of mental disorders (DSM-IV-TR) ${ }^{(15,16)}$. Os pesquisadores também registraram dados secundários obtidos dos prontuários dos pacientes idosos no intuito de complementar as informações relativas ao contexto da institucionalização.

A população do estudo consistiu de pessoas idosas institucionalizadas do Município de Fortaleza, Ceará, locus desta pesquisa, que possui 2.500 .094 habitantes, dos quais 242.430 pessoas com 60 anos e mais ${ }^{(17)}$. Conta com nove ILPI, sendo duas de pequeno porte, cinco de médio e duas de grande porte. Podem ter natureza governamental ou pública e não governamental (privada ou filantrópica). Neste estudo, optou-se por examinar todos os idosos das duas ILPI de grande porte pelo fato de abrigarem o maior número de idosos, configurando-se, portanto, em um censo. Nestas duas instituições são abrigados, em média, 310 idosos (210 na não governamental e 100 na governamental) de um total de 512 idosos institucionalizados no município em questão. Um total de 237 idosos participou da pesquisa.

Os critérios de inclusão eram: idosos residentes com idade de 60 anos e mais, que possuíam capacidade cognitiva para se submeter à entrevista na avaliação clínica e que quiseram participar do estudo, assinando o Termo de Consentimento Livre e Esclarecido.

Utilizou-se o programa SPSS Statistic versão 20 (SPSS Inc, Chicago, IL, USA), para o processamento, tabulação e análise dos dados, sendo as variáveis submetidas à análise descritiva e analítica. Para a análise univariada, calcularam-se frequências absoluta e relativa, e, na análise bivariada, os testes de qui- quadrado de Fischer e Pearson, considerando o nível de significância de 5\% e o intervalo de confiança de $95 \%$.

O projeto de pesquisa obteve aprovação pelo Comitê de Ética e Pesquisa da Universidade de Fortaleza, Parecer $n^{\circ} 148.718 / 2012$.

\section{RESULTADOS}

A idade dos idosos participantes da pesquisa variou de 60 a 104 anos, tendo como média 75,3 anos (DP $\pm 8,574$ ).

De acordo com a Tabela I, constatou-se que, do total de idosos pesquisados, $82(34,6 \%)$ apresentaram diagnóstico de Depressão Maior segundo os critérios do DSM-IV-TR. No diagnóstico de depressão por ILPI, mostrou significância estatística entre as duas ILPI $(p=0,042)$.

Houve significância estatística entre motivo da institucionalização e tipo de ILPI; 24 idosos (29,3\%) institucionalizaramse por vontade própria na ILPI não governamental, enquanto nove $(11 \%)$ chegaram à ILPI governamental abandonados por suas famílias $(p=0,001)$. Relativamente à visita de familiares, $40(48,8 \%)$ recebiam-nas na ILPI não governamental, enquanto apenas cinco $(6,1 \%)$ dos idosos deprimidos tinham contato com seus parentes na ILPI governamental $(p=0,002)$.

Quando comparados os motivos de institucionalização e a visita de familiares, os que receberam menos visitas foram os institucionalizados devido ao abandono familiar (18,3\%) e aqueles com suporte familiar insuficiente $(15,9 \%)$. Por outro lado, os que mais receberam visitas foram os institucionalizados por vontade própria $(25,6 \%)$. 
Na Tabela II apresentam-se dados relativos aos idosos com diagnóstico de depressão maior, mostrando idosos em situação de abandono familiar (32,9\%) como principal motivo de institucionalização, seguido dos que se institucionalizaram por vontade própria $(30,5 \%)$.

Verificou-se significância estatística em relação ao idoso morar na ILPI por vontade própria e receber visitas de familiares $(p=0,009)$.

Tabela I - Prevalência de depressão maior em idosos institucionalizados (n=237). Fortaleza, Ceará, 2016.

\begin{tabular}{lccccccc}
\hline \multirow{2}{*}{ Depressão } & \multicolumn{2}{c}{ ILPI } & \multicolumn{2}{c}{ ILPI } & \multicolumn{2}{c}{ Total } & \multirow{2}{*}{ Valor de $\mathbf{p}^{*}$} \\
\cline { 2 - 7 } & $\mathbf{n}=\mathbf{1 5 9}$ & $\mathbf{\%}$ & $\mathbf{n = 7 8}$ & $\mathbf{\%}$ & $\mathbf{n = 2 3 7}$ & $\mathbf{\%}$ & \\
\hline Sim & 62 & 39,0 & 20 & 55,6 & 82 & 34,6 & \multirow{2}{*}{0,042} \\
Não & 97 & 61,0 & 58 & 74,4 & 155 & 65,4 & \\
\hline
\end{tabular}

ILPI: Instituição de Longa Permanência para Idosos. *Qui quadrado de Pearson

Tabela II - Idosos deprimidos, motivo de institucionalização e apoio informal de visita por familiares ( $n=82)$. Fortaleza, Ceará, 2012.

\begin{tabular}{|c|c|c|c|c|c|c|c|}
\hline \multirow{3}{*}{ Motivo da institucionalização } & \multicolumn{4}{|c|}{ Recebe visita dos familiares } & \multirow{2}{*}{\multicolumn{2}{|c|}{ Total }} & \multirow{3}{*}{ Valor de $\mathbf{p}^{*}$} \\
\hline & \multicolumn{2}{|c|}{ Sim } & \multicolumn{2}{|c|}{ Não } & & & \\
\hline & $\mathbf{n}$ & $\%$ & $\mathbf{n}$ & $\%$ & $\mathrm{n}$ & $\%$ & \\
\hline Vontade própria & 21 & 25,6 & 4 & 4,9 & 25 & 30,5 & 0,009 \\
\hline Abandono Familiar & 12 & 14,6 & 15 & 18,3 & 27 & 32,9 & \\
\hline Maus tratos & 2 & 4,4 & 4 & 10,8 & 6 & 7,3 & \\
\hline Suporte familiar insuficiente & 9 & 11,0 & 13 & 15,9 & 22 & 26,8 & \\
\hline Outros & 1 & 1,2 & 1 & 1,2 & 2 & 2,4 & \\
\hline
\end{tabular}

*Exato de Fisher

\section{DISCUSSÃO}

A prevalência de depressão maior em idosos institucionalizados, segundo os critérios do DSM-IV-TR, apresentouse elevada no presente estudo. Contudo, pode se apresentar com menor prevalência do que em pesquisas que utilizam os instrumentos de rastreamento. Autores demonstram taxas mais elevadas como no estudo realizado em 14 lares para idosos em Bragança, onde há prevalência de 46,7\% de casos de depressão(18), constituindo motivo para maiores ações de promoção e prevenção em todos os níveis de atenção à saúde.

Em outro estudo realizado em ILPI de Goiânia, foi verificado um percentual de 40,7\% pela escala de Depressão geriátrica abreviada de Yesavage ${ }^{(19)}$. Idosos residentes em ILPI em Portugal apresentaram prevalência de 61,4\% de sintomas depressivos ${ }^{(20)}$. Resultados semelhantes foram verificados em Hong Kong e Taiwan onde se evidenciou uma prevalência de $65,4 \%$ e 43,4\% respectivamente, sendo esta diferença estatística demonstrada na pesquisa por pior status socioeconômico dos idosos de Hong Kong ${ }^{(21)}$. Essa diferença entre as prevalências pode ser explicada pela variabilidade no que concerne às formas de diagnóstico da depressão maior, os diferentes ambientes em que o idoso está inserido e o contexto clínico e social.

A prevalência de idosos com depressão foi maior nos solteiros e viúvos no presente estudo, sugerindo que a solidão ou luto pela morte de um cônjuge na senilidade pode ser fator de risco para desenvolvimento de sintomas depressivos. Um estudo(22) também mostrou que $46,7 \%$ dos idosos deprimidos eram solteiros, seguidos de $36,6 \%$ de viúvos e $16,7 \%$ de divorciados, assim como em pesquisa realizada em ILPI em Minas Gerais, onde $57 \%$ dos idosos eram solteiros ${ }^{(23)}$. Ressalta-se que em uma investigação realizada em cinco ILPI verificou-se prevalência de $49 \%$ de idosos com depressão pela Escala de Depressão Geriátrica de Yesavage, com predominância do sexo feminino $(60,8 \%)$ e sem cônjuge $(35,3 \%)^{(24)}$.

Em uma pesquisa realizada no Brasil, constituída por 11 ILPI públicas e privadas, a prevalência de sintomas depressivos foi de $55 \%$, sendo mais prevalente em idosos na faixa etária de 70-79 anos, sem cônjuge, com menos de 5 anos de escolaridade ${ }^{(25)}$, perfil semelhante ao encontrado na atual pesquisa. 
As variáveis como sexo feminino, doenças de etiologia somática, déficit funcional, neurossensorial e cognitivo, isolamento social, antecedente de depressão, solidão, viuvez, perda de entes queridos e a própria institucionalização, são considerados fatores desencadeadores de quadros depressivos, assim como falta de suporte social e familiar inadequados ${ }^{(26)}$.

A falta de cônjuge para assistir ao idoso pode contribuir para a ida e permanência definitiva em uma ILPI pela falta de alguém para supervisioná-lo em seus cuidados ${ }^{(22)}$. Da mesma forma, estudo conduzido em várias ILPI na China $^{(21)}$ demonstrou predominância de sintomas depressivos nos idosos institucionalizados com pior suporte social, incluindo a ausência de um companheiro.

Houve significância estatística entre motivo da institucionalização e tipo de ILPI na presente pesquisa; 24 idosos $(29,3 \%)$ institucionalizaram-se por vontade própria na ILPI não governamental, enquanto nove (11\%) chegaram à ILPI governamental abandonados por suas famílias $(p=0,001)$. Relativamente à visita de familiares, $40(48,8 \%)$ recebiam-nas na ILPI não governamental, enquanto apenas cinco $(6,1 \%)$ dos idosos deprimidos tinham contato com seus parentes na ILPI governamental $(p=0,002)$. Pesquisa em idosos institucionalizados evidenciou que o nível de depressão tende a ser maior nos idosos que não ingressaram no lar por iniciativa própria, sugerindo que a falta de apoio social e familiar contribui para a maior frequência de sintomas depressivos ${ }^{(27)}$.

$\mathrm{Na}$ associação verificada no atual estudo entre depressão e tempo de institucionalização $(p=0,001)$, é importante destacar o maior percentual entre os idosos com até três anos de institucionalização ( $37,8 \%$ dos casos de depressão), levando-se a considerar que o pouco tempo de afastamento familiar e o processo de adaptação ao novo tipo de moradia possam constituir fatores de risco para a doença. Este fato pode estar relacionado à significância estatística entre depressão e idosos que recebem visita de familiares $(p=0,036)$, cujo fato sugere que os vínculos familiares ainda perduram e se fortalecem a cada visita, tornando a separação mais intensamente penosa, fato este comprovado em pesquisa na qual $36,6 \%$ dos idosos que apresentavam sintomas depressivos estavam institucionalizados há menos de um ano(22).

No que tange ao apoio familiar, o grupo familiar funcional é capaz de responder a situações de conflito com certa estabilidade emocional, enquanto o disfuncional, por outro lado, irá prejudicar a pessoa em certas situações. Dessa forma, a qualidade familiar irá influenciar no status de saúde de forma significativa, de modo que a deterioração do suporte familiar acelera a incidência de sintomas depressivos, uma vez que pode provocar situações de solidão e sentimentos de abandono, demonstrado em estudo no qual os idosos com disfunção familiar eram 6,1 vezes mais propensos a apresentar sintomas depressivos ${ }^{(28)}$.

Paralelamente, outro fator que pode influenciar no surgimento de sintomas depressivos em idosos institucionalizados é o sentimento, por parte do paciente, de esperança de que sua família assumirá seu cuidado em determinado momento, principalmente aqueles que tiveram filhos ${ }^{(29)}$. Entretanto, pesquisas apontam que as visitas domiciliares diminuem em frequência à medida em que o tempo de institucionalização aumenta, demonstrando que os laços familiares se fragilizam com o passar do tempo, corroborando a piora dos sintomas depressivos por parte do idoso institucionalizado ${ }^{(30)}$.

A depressão é o transtorno psiquiátrico mais frequente na população idosa, sendo diagnosticada através dos critérios do DSM-IV-TR e gerando incapacidade e piora na qualidade de vida na velhice. Ademais, sua prevalência é ainda maior em idosos institucionalizados.

\section{CONCLUSÃO}

Os achados da pesquisa aludem à ideia de que o tempo de institucionalização, a carência das relações interpessoais, a solidão e o fato de o idoso receber visitas de familiares neste período constituem fatores de risco para a depressão, levando a considerar que os vínculos familiares perduram, fortalecendo-se a cada visita, portanto, tornando a ruptura familiar ainda mais penosa, o que compromete o direito do idoso de envelhecer com dignidade.

\section{CONFLITOS DE INTERESSE}

Autores informam que não há conflitos de interesse.

\section{AGRADECIMENTOS}

À Universidade de Fortaleza e à Diretoria de Pesquisa, Desenvolvimento e Inovação. 


\section{REFERÊNCIAS}

1. United Nations. Department of Economic and Social Affairs. World Population Ageing 2015 [Internet]. New York: United Nations; 2015. Disponível em: http://www.un.org/en/development/desa/population/

2. Yarahmadi SH, Etemad K, Hazaveh AM, Azhang N. Urbanization and Non-communicable risk factors in the capital city of 6 big provinces of Iran. Iran J Public Health. 2013;42(Suppl 1):113-8.

3. World Health Organization. Follow-up to the political declaration of the High-level Meeting of the General Assembly on the Prevention and Control of Non-communicable Diseases [Internet]. 2013; [acesso em: 2016 Jul 12]. Disponível em: http://apps.who.int/gb/ebwha/pdf_files/wha66/a66_r10-en.pdf

4. Chevreul K, Berg Brigham K, Bouché C. The burden and treatment of diabetes in France. Global Health. 2014;10(6):1-25. Doi: 10.1186/1744-8603-10-6.

5. Park SC, Lee HY, Lee DW, Hahn SW, Park SH, Kim YJ; et al. Screening for Depressive disorder in elderly patients with chronic physical diseases using the patient health. Questionnaire-9. Psychiatry Investig. 2017;14(3):306-13. Doi: 10.4306/pi.2017.14.3.306.

6. Frank C. Pharmacologic treatment of depression in the elderly. Can Fam Physician. 2014;60(2):121-6.

7. Ulbricht CM, Rothschild AJ, Hunnicutt JN, Lapane KL. Depression and cognitive impairment among newly admitted nursing home residents in the USA. Int J Geriatr Psychiatry. 2017;32:1172-1181. Doi:10.1002/gps.4723

8. Hagan R, Menktelow R, Taylor BJ, Mallett J. Reducing loneliness amongst older people: A systematic search and narrative review. Aging Ment Health 2014;18(6):683-693. Doi: 10.1080/13607863.2013.875122.

9. Van Beljouw IMJ, Van Exel E, De Jong GJ, Comijs HC, Heerings M, Stek ML, Van Marwijk. "Being all alone makes me sad": Loneliness in older adults with depressive symptoms. Int Psychogeriatr. 2014;1-11. Doi: 10.1017/ S1041610214000581.

10. Bodner E, Bergman YS. Loneliness and depressive symptoms among older adults: The moderating role of subjective life expectancy. Psychiatry res. 2016;237:78-82. Doi: 10.1016/j.psychres.2016.01.074.

11. Siqueira GR, Vasconcelos DT, Duarte GC, Arruda IC, Costa JAS, Cardoso RO. Análise da sintomatologia depressiva nos moradores do abrigo Cristo Redentor através da aplicação da escala de depressão geriátrica (EDG). Ciênc Saúde Colet. 2009;14(1):253-259.

12. Teston EF, Carreira L, Marcon SS. Sintomas depressivos em idosos: comparação entre residentes em condomínio específico para idoso e na comunidade. Rev Bras Enferm. 2014;67(3):450-456. Doi: 10.5935/0034-7167.20140060.

13. Martire LM, Schulz R. Involving Family in Psychosocial Interventions for Chronic Illness. Curr Dir Psychol Sci. 2007;16(2):90-94.

14. National Institutes of Health. Institute of Medicine. Health and Behavior: The interplay of biological, behavioral, and social influence. Washington: The national Academied Press; 2001.

15. American Psychiatric Association. Diagnostic and statistical manual of mental disorders: DSM-IV-TR. 4a ed. Washington: American Psychiatric Association; 2000. p. 463-72.

16. Thakur M, Blazer DG. Depression in Long-Term Care. J Am Med Dir Assoc. 2008;9(02):82-87.

17. Brasil. Ministério da Saúde. Departamento de Informática do Sistema Único de Saúde. População residente no Estado do Ceará [Intenet]. 2012; [acesso em 2017 Aug 31]. Disponível em: http://tabnet.datasus.gov.br/cgi/ tabcgi.exe?ibge/cnv/popce.def.

18. Vaz SFA, Gaspar NMS. Depressão em idosos institucionalizados no distrito de Bragança. Rev Enferm Referência. 2011;3(4):49-58.

19. Menezes RL, Bachion MM, Souza JT, Nakatani AYK. Estudo longitudinal dos aspectos multidimensionais da saúde de idosos institucionalizados. Rev Bras Geriatr Gerontol. 2011;14(3):485-96.

20. Leal MCC, Apostolo JLA, Mendes AMOC, Marques APO. Prevalence of depressive symptoms and associated factors among institutionalized elderly. Acta Paul Enferm. 2014;27(3):208-14. 
21. Tsai YF, Chung JW, Wong TK, Huang CM. Comparison of the prevalence and risk factors for depressive symptoms among elderly nursing home residents in Taiwan and Hong Kong. Int J Geriat Psychiatry. 2005;20:(4)315-21.

22. Carreira L, Botelho MR, Matos PCB, Torres MM, Salci MA. Prevalência de depressão em idosos institucionalizados. Rev Enferm UERJ. 2011;19(2):268-73.

23. Galhardo VAC, Mariosa MAS, Takata JPI. Depressão e perfis sociodemográfico e clínico de idosos institucionalizado sem déficit cognitivo. Rev Méd Minas Gerais. 2010;20(1)16-21.

24. Silva ER, Sousa ARP, Ferreira LB, Peixoto HM. Prevalence and factors associated with depression among institutionalized elderly individuals: nursing care support. Rev Esc Enferm USP. 2012;46(6):1387-93.

25. Güths JFS, Jacob MHVM, Santos AMPV, Arossi GA, Béria JU. Perfil sociodemográfico, aspectos familiares, percepção de saúde, capacidade funcional e depressão em idosos institucionalizados no Litoral Norte do Rio Grande do Sul, Brasil. Rev Bras Geriatr Gerontol. 2017;20(2):175-85.

26. Nóbrega IRAP, Leal MCC, Marques APO, Vieira JCM. Fatores associados à depressão em idosos institucionalizados: revisão integrativa. Saúde Debate. 2015;39(105):536-50.

27. Pimentel AF, Afonso RM, Pereira H. Depression and social support in old age. Psicol Saúde Doenças. 2012;13(2): 311-27.

28. Oliveira SC, Santos AA, Pavarini SCI. The relationship between depressive symptoms and family functioning in institutionalized elderly. Rev Esc Enferm USP. 2014;48(1):6-72.

29. Silva LR, Vázquez-Garnica EK, Karina E. O cuidado aos idosos: considerações sobre o cuidado não familiar. Texto \& contexto enferm. 2008;17(2):225-31.

30. Perlini NMOG, Leite MT, Furini AC. Em busca de uma instituição para a pessoa idosas morar: motivos apontados por familiares. Rev Esc Enferm USP. 2007;41(2):229-36.

\section{Endereço para correspondência:}

Maria Vieira de Lima Saintrain

Universidade de Fortaleza - UNIFOR

Programa de Pós-Graduação em Saúde Coletiva

Av. Washington Soares, 1321, Bloco S, Sala 1

Bairro: Edson Queiroz

CEP: 60.811-905 - Fortaleza - CE - Brasil

E-mail: mariavieira@bol.com.br 